\title{
ON BINARY SEQUENCES ${ }^{1}$
}

\section{R. TURYN AND J. STORER}

Let $x_{i}= \pm 1$, tor $i=1, \cdots, n$ and let $c_{k}=\sum_{1}^{n-k} x_{i} x_{i+r}$. We shall be concerned with sequences $\left(x_{i}\right)$ for which $\left|c_{k}\right| \leqq 1,0<k<n$. The problem was first considered in [1]. The following sequences with this property are known:

$$
\begin{aligned}
n=2 & ++ \\
3 & ++- \\
4 & +++-;++-+ \\
5 & +++-+ \\
7 & +++--+- \\
11 & +++---+-+- \\
13 & +++++--++-+-+
\end{aligned}
$$

where it has been assumed $x_{1}=x_{2}=+1$. Each one of these gives rise to three others under the transformations

$$
\begin{aligned}
& x_{i}^{\prime}=(-1)^{i} x, \\
& x_{i}^{\prime}=(-1)^{i+1} x_{i}, \\
& x_{i}^{\prime}=-x_{i} .
\end{aligned}
$$

The above sequences have the following interpretation [2]: the elements which are -1 are those with subscript given $\bmod n$ by

$$
\begin{aligned}
& n=5: \quad m^{4}+3, \quad m \neq \equiv 0(\bmod 5), \\
& n=7: \quad m^{2}+3, \quad m \neq \equiv(\bmod 7), \\
& n=11: \quad-m^{2}-2, \quad(\bmod 11) \text {, } \\
& n=13: \quad 11 m^{4}-1, \quad(\bmod 13) \text {. }
\end{aligned}
$$

It will be shown here that for odd $n$, these are all the sequences with the property $\left|c_{k}\right| \leqq 1$. For the sake of completeness, the preliminary details have been included.

Let $\left(x_{i}\right)$ be any sequence with $x_{i}= \pm 1$, and let

Received by the editors May 26, 1960.

1 The research reported in this paper has been partially sponsored by the Electronics Research Directorate of the AFCRC, Air Research and Development Command, under Contract AF-19(604)-5473. 


$$
c_{k}=\sum_{1}^{n-k} x_{i} x_{i+k}
$$

Since the terms $x_{i} x_{i+k}$ are each \pm 1 , it follows that:

$$
\prod_{1}^{n-k}\left(x_{i} x_{i+k}\right)=(-1)^{\left(n-k-c_{k}\right) / 2}, \quad 0 \leqq k<n .
$$

Multiplying two successive equations of this form gives

$$
x_{n-k} x_{k+1}=(-1)^{n-k-\left(1+c_{k}+c_{k+1}\right) / 2}, \quad 1 \leqq k \leqq n-1 .
$$

Now consider

$$
c_{k}+c_{n-k}=\sum_{1}^{n} x_{i} x_{i+k}
$$

(the second index being taken $\bmod n$ ). Then (2) yields

$$
\prod_{1}^{n}\left(x_{i} x_{i+k}\right)=1=(-1)^{\left(n-c_{k}-c_{n-k}\right) / 2}
$$

Thus $c_{k}+c_{n-k} \equiv n(\bmod 4)$.

We now assume $\left|c_{k}\right| \leqq 1,0<k<n$; thus $c_{k}=0$ for $n-k$ even, $c_{k}= \pm 1$ for $n-k$ odd. ${ }^{2}$ Now assume also that $n$ is odd. In this case, one of $c_{k}$ and $c_{n-k}$ is odd and the other case even, hence 0 . Thus

$$
\begin{aligned}
c_{2 i+1} & =0, \\
c_{2 j} & =(-1)^{(n-1) / 2},
\end{aligned}
$$

the latter since $c_{k}+c_{n-k} \equiv n(\bmod 4)$. The formula for $x_{n-k} x_{k+1}$ becomes

$$
x_{n-k} x_{k+1}=(-1)^{(n-1) / 2+k} \text {. }
$$

A sequence which satisfies (3) has the property that $c_{2 j+1}=0,0<$ $2 j+1<n$.

$$
c_{2 j}=\sum_{1}^{n-2 j} x_{i} x_{i+2 j}, \quad 0<2 j<n,
$$

or

$$
(-1)^{(n-1) / 2}=\sum_{1}^{n-2 j} x_{i} x_{n-2 j+1-i}(-1)^{(n-1) / 2+i+1} .
$$

Let $n-2 j=2 k+1, k \geqq 1$.

2 It follows from this that $n \equiv 2(\bmod 4)$ implies $n=2$. For if $n>2, c_{2}=c_{n-2}=0$, $c_{2}+c_{n-2}=0 \not \equiv 2(\bmod 4)$. Thus $n$ even, $n>2$ implies $n \equiv 0(\bmod 4), c_{k}+c_{n-k}=0$. 


$$
\begin{aligned}
1 & =\sum_{1}^{2 k+1} x_{i} x_{2 k+2-i}(-1)^{i+1} \\
& =2 \sum_{1}^{k} x_{i} x_{2 k+2-i}(-1)^{i+1}+x_{k+1}^{2}(-1)^{k+2}, \quad n>2 k+1 \geqq 3 .
\end{aligned}
$$

Hence

$$
\frac{1+(-1)^{k+1}}{2}=\sum_{1}^{k} x_{i} x_{2 k+2-i}(-1)^{i+1} \quad 1 \leqq k<\frac{n-1}{2} .
$$

We shall write $P(k)$ for $\left(1+(-1)^{k+1}\right) / 2$.

TheOREM 1. Let $\left(x_{i}\right)$ satisfy equations $(\mathrm{k})$ for $1 \leqq k \leqq t, x_{i}= \pm 1$. Let $x_{i}=1$ for $1 \leqq i \leqq p, x_{p+1}=-1$; assume $p>1$. Then

(i) $x_{i} x_{i+1}=x_{2 i} x_{2 i+1}, 1 \leqq i \leqq t$,

(ii) $p \leqq 2 t+1$ implies $p$ is odd,

(iii) $p j+r \leqq 2 t+1,1 \leqq r \leqq p$ implies $x_{p(j-1)+r}=x_{p(j-1)+1}$, and

(iv) $z_{j}=x_{p(j-1)+1}$ satisfy equations (k) for $k \leqq t / p$.

Proof. A count of +1 's and -1 's in equation ( $k$ ) shows as before that

$$
\prod_{1}^{k}\left(x_{i} x_{2 k+2-i}\right)=1
$$

or

$$
\prod_{1}^{2 k+1} x_{i}=x_{k+1}
$$

Multiplying two successive equations of this form proves the first statement. The second statement follows from the first: for if $p>2$ were even, say $p=2 s$, we would have

$$
x_{8} x_{s+1}=x_{2 s} x_{20+1}
$$

or, by assumption, $1=-1$.

We prove (iii) and (iv) by induction on $t$. From $t<p$, there is nothing to prove. For $p=t$, we have:

$$
1=x_{1} x_{2 p+1}-x_{2} x_{2 p}+\cdots+x_{p} x_{p+2} .
$$

Now $x_{i}=1$ for $i \leqq p$, and by the first part of the theorem we have $x_{2 k}=x_{2 k+1}$ for $p<2 k<2 p$. Thus the above equation reduces to

$$
1=x_{2 p+1}-x_{2 p}+x_{p+2} \text {. }
$$

But also 


$$
-1=x_{p} x_{p+1}=x_{2 p} x_{2 p+1}
$$

so we have

$$
1=2 x_{2 p+1}+x_{p+2}
$$

Thus

$$
\begin{aligned}
x_{p+2} & =-1, \\
x_{2 p+1} & =1 .
\end{aligned}
$$

Similarly, for $2 t+1 \leqq 3 p$, we let $t=p+s, s>0$; we have

$$
P(p+s)=\sum_{1}^{2 e+1}+\sum_{2 s+2}^{p}+\sum_{p+1}^{p+s}=x_{2 p+1}-x_{p+1} x_{p+2 o+1}+P(s-1),
$$

using the fact that $x_{2 i}=x_{2 i+1}$ for $i \neq 0(\bmod p)$ by the induction assumption and (i). Since $p$ is odd, $P(p+s)=P(s-1)$, and

$$
x_{2 p+1}+x_{p+2 q+1}=0
$$

or

$$
x_{p+20+1}=-1 \text {. }
$$

For $2 t+1>3 p$, we let $2 t+1=h p+m$ with $1 \leqq m \leqq p$, and consider two cases:

(A) $h$ odd, $h=2 H+1$. We have

$$
\sum_{1}^{t}=\sum_{0}^{H-1}\left[\sum_{j p+1}^{i p+m}+\sum_{j p+m+1}^{(j+1) p}\right]+\sum_{H p+1}^{H p+m}+\sum_{H p+m+1}^{t} .
$$

As before, we reduce the first three sums by using the fact that $x_{2 i}=x_{2 i+1}$ for $i \neq 0(\bmod p)$; the subsequent sums are of the form $(-1)^{i}$ by the induction assumption. Thus we get

$$
\begin{aligned}
P(t)= & x_{p} x_{(h-1) p+m+1}-x_{p+1} x_{(h-1) p+m}+x_{p+m} x_{(h-1) p+1} \\
& +\sum_{2}^{B} z_{i} z_{h+1-i}(-1)^{i+1}+(-1)^{B} P\left(\frac{p+m-1}{2}\right) .
\end{aligned}
$$

We have $t=H p+(p+m-1) / 2$. Therefore

$$
P(H)-\sum_{1}^{B} z_{i} z_{h+1-i}(-1)^{i+1}=x_{(h-1) p+m+1}+x_{(h-1) p+m}-2 x_{(h-1) p+1} .
$$

By the induction assumption, the left-hand side vanishes, and thus

$$
x_{(h-1) p+m}=x_{(h-1) p+1} \text {. }
$$

(B) If $h$ is even, $h=2 H$, we get, proceeding as before, 


$$
P(H)-\sum_{1}^{H} z_{i} z_{h+1-i}(-1)^{i+1}=-z_{h}-x_{h p}+2 x_{(h-1) p+m}
$$

for $m<p$. Subtracting successive equations of this type (i.e., $m=1,3, \cdots)$ gives $x_{(h-1) p+1}=x_{(h-1) p+m}$ (for $m$ odd, and the equation for $m$ even follows from (ii)). For $m=p$, i.e., $2 t+1=(2 H+1) p$ we get

$$
P(H)-\sum_{1}^{H} z_{i} z_{h+1-i}(-1)^{i+1}=z_{h}+x_{h p} .
$$

Comparing this with the equation for $m=1$, we deduce

$$
P(H)-\sum_{1}^{H} z_{i} z_{h+1-i}(-1)^{i+1}=0
$$

and hence

$$
x_{h p}=x_{(h-1) p+1} .
$$

We have shown that a sequence of odd length with $\left|c_{k}\right| \leqq 1$ for $k>0$ has some periodic behavior. We now conclude that it must therefore be short.

Theorem 2. Let $x_{i}= \pm 1,1 \leqq i \leqq n, n$ odd. Assume $\left|c_{k}\right| \leqq 1$ for $k>0$. Then $n \leqq 13$.

Proof. By using the transformations (1), we may assume that $x_{1}=x_{2}=1$. We have equations $(\mathrm{k})$ for $1 \leqq k<(n-1) / 2$, and clearly $p$ (as defined in Theorem 1) is less than $n-1$ for $n>3$. First, we must have $n<4 p$ : for if $n>4 p$, we would conclude by Theorem 1 that $x_{i}=+1,1 \leqq i \leqq p, 2 p+1 \leqq i \leqq 3 p$ and $x_{i}=-1, p+1 \leqq i \leqq 2 p$. But if $x_{i}=x_{i+1}, x_{n+1-i}=-x_{n-i}$ by (3), and runs of equal $x_{i}$ are opposite runs of alternating ones. $n>4 p$ would imply 3 initial constant blocks, hence 3 final alternating blocks, and therefore $n \geqq 6 p-1$. Similarly, for $k \geqq 2$ Theorem 1 shows that $n>k p$ implies $n \geqq 2(k-1) p-3$. Thus $n<4 p$. We also know $n \neq 3 p$, for $n=3 p$ would imply

$$
x_{p} x_{p+1}=x_{2 p} x_{2 p+1}=-x_{p+1} x_{p} \text {. }
$$

Let $a=(-1)^{(n-1) / 2}, n-3=2 m$. Then equation $(\mathrm{m})$ is

$$
\frac{1+a}{2}=\sum_{1}^{m} x_{i} x_{n-1-i}(-1)^{i+1} \text {. }
$$

The first $p-2$ terms in the sum are equal to $a$. Let $N$ be the number of terms $x_{i} x_{n-1-i}(-1)^{i+1}=-a$ with $p-2<i \leqq m$. Then 


$$
\begin{aligned}
& \frac{1+a}{2}=(p-2) a+(m-N-(p-2)) a-N a, \\
& \frac{a+1}{2}+N-(p-2)=m-N-(p-2) \geqq 0, \\
& p-2-\frac{a+1}{2} \leqq N \leqq \frac{n-3}{2}-(p-2),
\end{aligned}
$$

or

$$
4 p \leqq n+6+a .
$$

Hence $n<3 p$ implies $p<6+a$ or $p \leqq 5$. By Theorem 1 , we can construct the sequences of lengths $5,7,13$. Finally, for $3 p<n<4 p$, let $n=2 m+1 . x_{m}=-x_{m+2}$, and we have $x_{i}=-1$ for $p+1 \leqq i \leqq 2 p$. By Theorem 1 , we must have $n-2-p \leqq m+1=(n+1) / 2$, or $n \leqq 2 p+5$. Now $n>3 p$ implies $p<5$, and hence $p=3$ and we reconstruct the sequence of length 11 .

\section{REFERENCES}

1. R. H. Barker, Group synchronizing of binary digital systems, in Communication theory, London, 1953, pp. 273-287.

2. Marshall Hall, Jr., A survey of difference sets, Proc. Amer. Math. Soc. vol. 7 (1956) pp. 975-986.

Sylvania Electric Products, Inc. 